\title{
Application of Tsallis Cross-entropy in Image Thresholding Segmentation
}

\author{
Qian-Qian Lin, ${ }^{1}$ Ling Zhang, ${ }^{1}$ Tung-Lung Wu, ${ }^{2 *}$ \\ Tean-Shine Mean, ${ }^{3}$ and Hsien-Wei Tseng ${ }^{1 * *}$
}

\footnotetext{
${ }^{1}$ School of Mathematics and Information Engineering, Longyan University, Longyan 364012, Fujian, China ${ }^{2}$ School of Mechanical and Automotive Engineering, Zhaoqing University, Zhaoqing 516260, Guangdong, China ${ }^{3}$ International Institute of Knowledge Innovation and Invention (IIKII) Pte. Ltd., Singapore
}

(Received January 6, 2020; accepted July 8, 2020)

Keywords: image segmentation, Tsallis cross-entropy, long-range correlation

We propose a novel Tsallis cross-entropy thresholding method based on the premise that local long-range correlations rather than global long-range correlations may exist among the gray levels of pixels. The target and background of the image can be considered as two independent parts, and their information integrity can be maximized by the proposed method. Our experimental results show that this method can obtain better segmentation results than the minimum Tsallis cross-entropy thresholding method when considering global long-range correlations when segmenting images in which the object and background have no obvious correlations.

\section{Introduction}

The minimum Tsallis cross-entropy thresholding method minimizes the difference in the amount of information between an original image and a segmented image so as to guarantee that the computer reading of the internal information of the image is as faithful as possible to the original image. In 1993, Li and Lee defined cross-entropy and applied it in the study of image threshold segmentation. ${ }^{(1)}$ Subsequently, the research groups of Brink and Pal have continuously improved this method, which has greatly expanded its range of applications. ${ }^{(2,3)}$ In China, there have also been many studies in this area. For example, Xue and Zhang analyzed the probability distribution of images after mixed Poisson distribution estimation and proposed a method to estimate the probability distribution of a segmented image with a mixed uniform distribution. $^{(4)}$ They also proposed a maximum interclass cross-entropy criterion function so that the target and background of the image can be separated as much as possible. Tang and coworkers proposed a segmentation method based on the minimum Tsallis cross-entropy threshold and a two-dimensional minimum Tsallis cross-entropy threshold segmentation method. $(5,6)$ Note that the introduction of Tsallis entropy in statistical physics is based on the

\footnotetext{
*Corresponding author: e-mail: tunglung216@gmail.com

** Corresponding author: e-mail: hsienwei.tseng@gmail.com https://doi.org/10.18494/SAM.2020.2798
} 
experimental findings of long-range interactions within physical systems. ${ }^{(7,8)}$ In terms of image processing, this long-range effect can be understood as the correlation between the gray values of each pixel inside an image. ${ }^{(9-11)}$ This correlation, which is different from the correlation between the pixels in the domain mentioned above, is global. However, since there are various image categories, not all the internal pixel gray value correlations of an image are global.

In an infrared target recognition system, the infrared image sequence obtained by an infrared sensor has a certain relationship with the temperature distribution of each point on the surface, which results in a correlation between the brightness values of the pixels in the target, but the temperature distributions of the target and background are not necessarily related. Here, we propose a novel Tsallis cross-entropy thresholding method based on the premise to deal with images with no obvious correlation between the target and the background.

\section{Principle of Minimum Tsallis Cross-entropy Thresholding Method}

\subsection{Tsallis cross-entropy}

Furuichi et al. proposed the concept of Tsallis cross-entropy in 2004 and defined the Tsallis correlated entropy between two probability distributions $P=\left\{p_{1}, p_{2}, \ldots, p_{N}\right\}$ and $Q=\left\{q_{1}, q_{2}, \ldots, q_{N}\right\}$ as

$$
D_{q}(P, Q)=-\sum_{i=1}^{N} p_{i} \frac{1-\left(\frac{p_{i}}{q_{i}}\right)^{1-q}}{q-1}
$$

where $q$ is a non-negative real number. ${ }^{(12)}$ When $q \rightarrow 1$, the Tsallis cross-entropy is converted into the form of the traditional cross-entropy, that is,

$$
\lim _{q \rightarrow 1} D_{q}(P, Q)=\lim _{q \rightarrow 1}\left(\sum_{i=1}^{N} p_{i} \frac{\left(\frac{p_{i}}{q_{i}}\right)^{1-q}-1}{q-1}\right)=\sum_{i=1}^{N} p_{i} \ln \frac{p_{i}}{q_{i}} .
$$

The symmetry form of the Tsallis cross-entropy can be defined as

$$
D_{q}(P: Q)=\sum_{i=1}^{N} p_{i} \frac{\left(\frac{p_{i}}{q_{i}}\right)^{1-q}-1}{q-1}+\sum_{i=1}^{N} q_{i} \frac{\left(\frac{q_{i}}{p_{i}}\right)^{1-q}-1}{q-1} .
$$

\subsection{Minimum Tsallis cross-entropy thresholding method}

We set the probability distribution $P=\left\{p_{1}, p_{2}, \ldots, p_{N}\right\}$ as the gray-level histogram probability distribution of the original image and the probability distribution $Q=\left\{q_{1}, q_{2}, \ldots, q_{N}\right\}$ as the 
gray-level histogram probability distribution of the thresholded image. Suppose that the threshold $t$ divides the original image into two parts, namely, the target and background, where pixels whose gray level is smaller than $t$ constitute the target region (represented by $O$ ) and pixels whose gray level is larger than $t$ constitute the background region (represented by $B$ ). From Eq. (3), the Tsallis cross-entropy between pixels within the target region is

$$
D_{q}^{O}(t)=\sum_{i=0}^{t} p_{i} \frac{\left(\frac{p_{i}}{q_{i}}\right)^{1-q}-1}{q-1}+\sum_{i=0}^{t} q_{i} \frac{\left(\frac{p_{i}}{q_{i}}\right)^{1-q}-1}{q-1}
$$

The Tsallis cross-entropy between pixels within the background region is

$$
D_{q}^{B}(t)=\sum_{i=t+1}^{n} p_{i} \frac{\left(\frac{p_{i}}{q_{i}}\right)^{1-q}-1}{q-1}+\sum_{i=t+1}^{n} q_{i} \frac{\left(\frac{p_{i}}{q_{i}}\right)^{1-q}-1}{q-1}
$$

where $q$ is a non-extensive parameter, which represents the correlation between the target and the background in the image. $n$ is the upper limit of the gray level of the image. The gray-level histogram probability distribution of the segmented image $\left\{q_{i}\right\}$ is estimated using the mixed uniform distribution proposed by Xue and Zhang, ${ }^{(4)}$ that is,

$$
q_{i}=\left\{\begin{array}{lc}
\frac{P_{O}}{t+1} & 0 \leq i \leq t \\
\frac{P_{B}}{n-t-1} & t+1 \leq i \leq n
\end{array} .\right.
$$

The Tsallis cross-entropy between $P$ and $Q$ in the image thresholding is defined as the sum of the Tsallis cross-entropy between pixels within the target region and the Tsallis cross-entropy between pixels within the background region. Then, according to the non-extensive property of the Tsallis entropy, the total Tsallis cross-entropy of the image before or after segmentation can be obtained as

$$
D_{q}(P: Q ; t)=D_{q}^{O}(t)+D_{q}^{B}(t)+(1-q) D_{q}^{O}(t) D_{q}^{B}(t) .
$$

When $D_{q}(P: Q ; t)$ takes a minimum value, the corresponding gray value is the optimal threshold value, ${ }^{(5)}$ that is,

$$
T_{o p t}=\underset{0 \leq t \leq n}{\arg \min }\left[D_{q}(P: Q ; t)\right] .
$$




\section{New Tsallis Cross-entropy Thresholding Method}

For some images whose targets can be changed arbitrarily, it has been a priori judged that there is no correlation between the target and the background; however, there may be a correlation within the target or within the background. That is, there is only a local longrange correlation in the image rather than a global long-range correlation. Therefore, under the assumption of global relevance, the minimum Tsallis cross-entropy thresholding method is used to segment these images, which could cause an intelligent algorithm to analyze the images in the wrong direction. In such a case, the information contained in the image cannot be understood correctly by a computer, and the information error between the obtained results and the original image cannot be minimized. Thus, we propose a new Tsallis cross-entropy thresholding method by modifying the minimum Tsallis cross-entropy thresholding method.

The criterion for the proposed thresholding algorithm is

$$
T_{o p t}=\underset{0 \leq t \leq n}{\arg \min }\left\{\max \left[D^{O}(t), D_{q}^{B}(t)\right]\right\},
$$

where $D^{O}(t)=\sum_{i=0}^{t} p_{i} \ln \frac{p_{i}}{q_{i}}+\sum_{i=0}^{t} q_{i} \ln \frac{q_{i}}{p_{i}}$ is the limit of Eq. (4) when $q \rightarrow 1$ and $D_{q}^{B}(t)$ is given by Eq. (5). Since the correlation between the gray levels of the pixels in the target region of some test images is weak and the correlation between the gray levels of the pixels in the background area is strong, it is clearly inappropriate to use the same $q$ value to describe the correlation strengths of the target and background. From a low correlation strength, it can be inferred that the value of the non-extensive parameter is close to 1 , so the cross-entropy defined by Kullback is adopted to describe the difference between the information in the target region before and after the segmentation. In contrast, for the background region, since the correlation between the pixel gray levels is stronger, the form of the Tsallis cross-entropy is retained and the correlation strength can be described by the $q$ value. On the other hand, for images of this type, at present, there is still insufficient evidence to show that the target and background must have long-range correlations, so we neglect the third term in Eq. (7). To achieve the objective of minimizing the cross-entropy value, the best approach should be to minimize the target and background cross-entropies at the same time. Generally, it is not easy to minimize the target and background cross-entropies using a threshold value. Therefore, the equilibrium value is substituted into Eq. (9), that is, the minimum value of the larger one. This constraint guarantees that both cross-entropies are smaller and as close as possible to the preset optimal criterion.

\section{Experimental Results and Discussion}

To verify the validity of the proposed method, some images are tested using the method and the results are compared with those obtained by the minimum Tsallis cross-entropy thresholding method. ${ }^{(5)}$ Four images are used: potatoes $(256 \times 256)$, coins with a black background $(300 \times 246)$, coins with a white background $(308 \times 242)$, and rice $(256 \times 256)$. 
Table 1 shows the threshold values obtained by the proposed and minimum Tsallis crossentropy thresholding methods for various $q$ values, where Threshold 1 is the threshold value obtained by the minimum Tsallis cross-entropy thresholding method and Threshold 2 is the threshold value obtained by the proposed method. It can be seen clearly from the table that the threshold value obtained by the minimum Tsallis cross-entropy thresholding method is poor and the parameter $q$ cannot perform the regulation function well. Changes in $q$ have little effect on the threshold, while the threshold value obtained by the proposed method responds sensitively to $q$.

To quantize the segmentation quality of various methods, we adopt a common image segmentation quality evaluation criterion, the misclassification error $(M E)$, to evaluate the quality of the segmented images. ${ }^{(13)}$ This criterion is a measure of the quality of image segmentation based on the consistency of pixels between the segmented and reference images. For the problem of single-threshold segmentation, $M E$ can be expressed simply as

$$
M E=1-\frac{\left|B_{G T} \cap B_{T}\right|+\left|F_{G T} \cap F_{T}\right|}{\left|B_{G T}\right|+\left|F_{G T}\right|},
$$

where $B_{G T}$ and $B_{T}$ are the sets of background pixels of the reference and thresholded images, and $F_{G T}$ and $F_{T}$ are the sets of target pixels of the reference and thresholded images, respectively, and the symbol $|\cdot|$ is the number of elements in the set. $M E$ is the percentage of target pixels misclassified as background pixels or the percentage of background pixels misclassified as target pixels. It can be seen from Table 1 that the misclassification error (ME2) obtained by the proposed method is smaller than the misclassification error (ME1) obtained by the

Table 1

Threshold results and MEs of test images for various $q$ values.

\begin{tabular}{|c|c|c|c|c|c|c|}
\hline Test image & $q=0.5$ & $q=0.6$ & $q=0.7$ & $q=0.8$ & $q=0.9$ & $q=1.0$ \\
\hline \multicolumn{7}{|c|}{ Potatoes } \\
\hline Threshold1 & 1 & 1 & 1 & 1 & 1 & 1 \\
\hline ME1 & 0.62784 & 0.62784 & 0.62784 & 0.62784 & 0.62784 & 0.62784 \\
\hline Threshold2 & 255 & 255 & 86 & 65 & 65 & 66 \\
\hline ME2 & 0.37193 & 0.37193 & 0.0054626 & 0.024307 & 0.024307 & 0.021378 \\
\hline \multicolumn{7}{|c|}{ Coins } \\
\hline Threshold1 & 255 & 255 & 255 & 255 & 255 & 232 \\
\hline ME1 & 0.32734 & 0.32734 & 0.32734 & 0.32734 & 0.32734 & 0.31493 \\
\hline Threshold2 & 78 & 78 & 78 & 78 & 78 & 48 \\
\hline ME2 & 0.00084011 & 0.00084011 & 0.00084011 & 0.00084011 & 0.00084011 & 0.6656 \\
\hline \multicolumn{7}{|c|}{ Eight } \\
\hline Threshold1 & 255 & 255 & 255 & 255 & 255 & 255 \\
\hline ME1 & 0.71937 & 0.71937 & 0.71937 & 0.71937 & 0.71937 & 0.71937 \\
\hline Threshold2 & 217 & 96 & 88 & 79 & 73 & 235 \\
\hline ME2 & 0.0040651 & 0.1841 & 0.22737 & 0.26322 & 0.27467 & 0.71559 \\
\hline \multicolumn{7}{|c|}{ Rice } \\
\hline Threshold1 & 118 & 118 & 118 & 118 & 118 & 118 \\
\hline ME1 & 0.025925 & 0.025925 & 0.025925 & 0.025925 & 0.025925 & 0.025925 \\
\hline Threshold2 & 93 & 87 & 122 & 122 & 122 & 121 \\
\hline ME2 & 0.39384 & 0.44244 & 0.013519 & 0.013519 & 0.013519 & 0.015854 \\
\hline
\end{tabular}


minimum Tsallis cross-entropy thresholding method, that is, the quality of the segmentation image obtained by the proposed method is superior, which can also be seen from the images. In Figs. 1-4, (d) and (e) respectively show the optimal segmentation effects of the above four test images by the proposed and minimum Tsallis cross-entropy thresholding methods. For the images of potatoes, coins, and rice, the proposed method effectively segments the target from

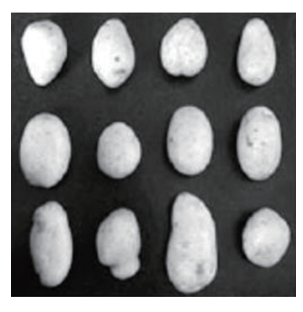

(a)

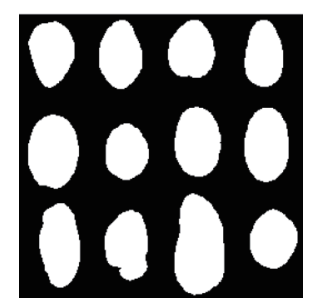

(b)

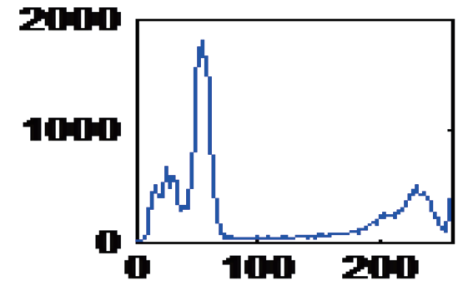

(c)

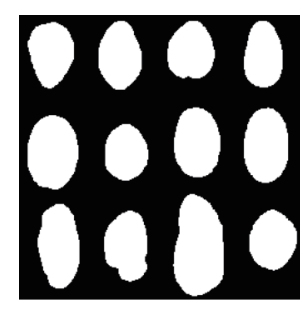

(d)

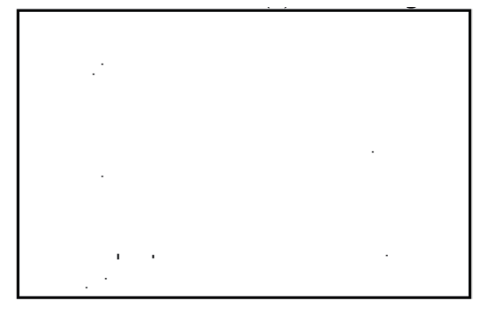

(e)

Fig. 1. (Color online) Image of potatoes and its optimal segmentation results. (a) Original image, (b) ground truth image, (c) ID histogram, (d) proposed method $(q=0.7)$, and (e) minimum Tsallis cross-entropy thresholding method $(q=0.7)$.

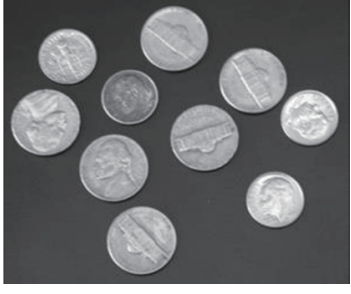

(a)

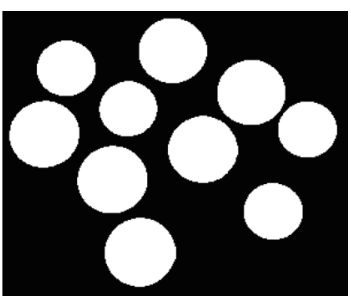

(b)

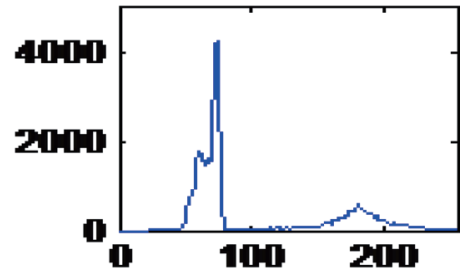

(c)

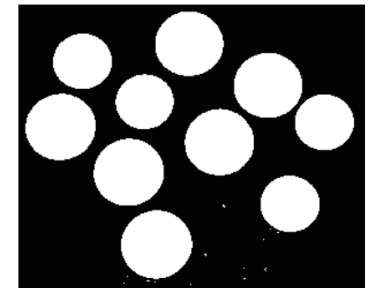

(d)

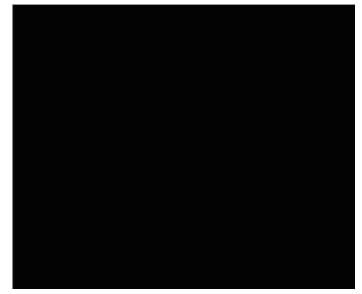

(e)

Fig. 2. (Color online) Image of coins with black background and its optimal segmentation results. (a) Original image, (b) ground truth image, (c) ID histogram, (d) proposed method ( $q=0.7$ ), and (e) minimum Tsallis cross-entropy thresholding method $(q=0.7)$. 


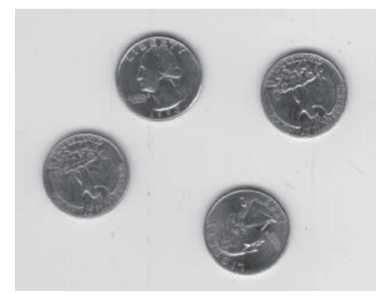

(a)

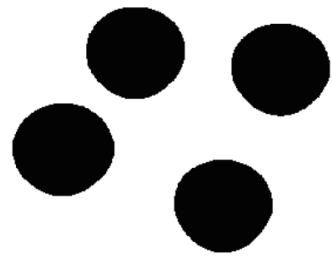

(b)

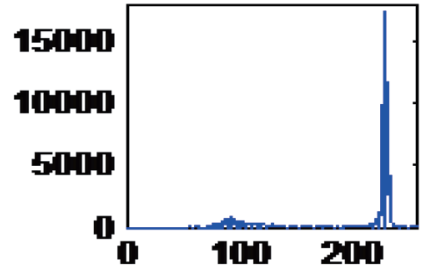

(c)

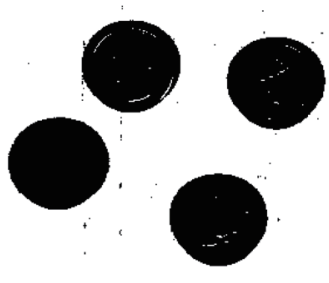

(d)

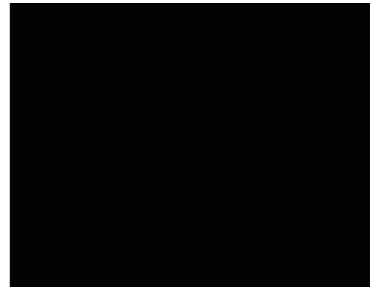

(e)

Fig. 3. (Color online) Image of coins with white background and its optimal segmentation results. (a) Original image, (b) ground truth image, (c) ID histogram, (d) proposed method ( $q=0.5$ ), and (e) minimum Tsallis cross-entropy thresholding method $(q=0.5)$.

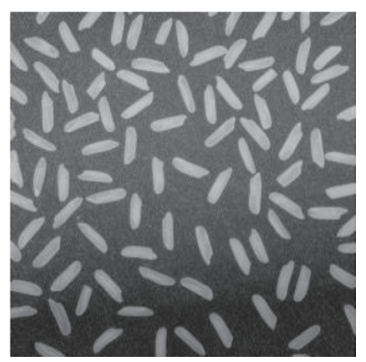

(a)

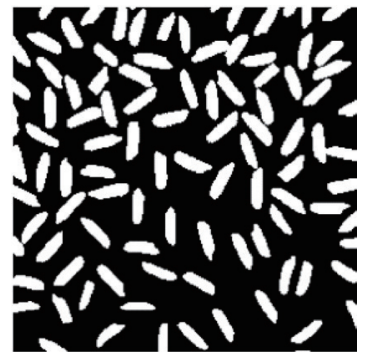

(b)

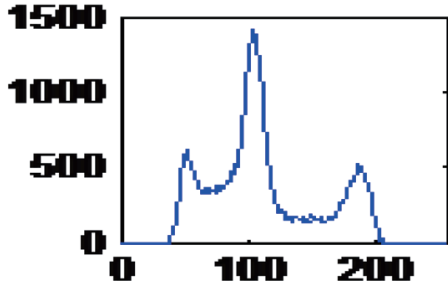

(c)

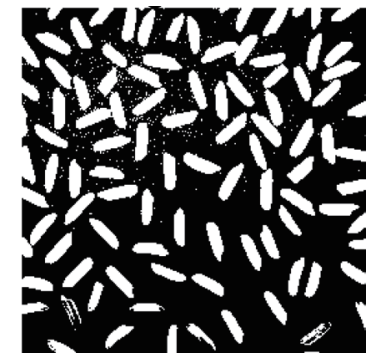

(d)

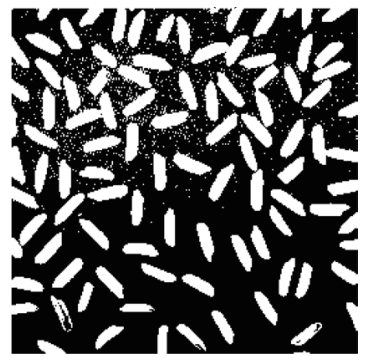

(e)

Fig. 4. (Color online) Image of rice and its optimal segmentation results. (a) Original image, (b) ground truth image, (c) ID histogram, (d) proposed method $(q=0.7)$, and (e) minimum Tsallis cross-entropy thresholding method $(q=0.7)$.

the background, in contrast to the minimum Tsallis cross-entropy thresholding method. From the image of rice in Fig. 4, it can be seen that the segmentation result obtained by the proposed method has less noise and the outline of the target is much clearer. 
Compared with the traditional Shannon entropy thresholding method, the maximum Tsallis entropy and minimum Tsallis cross-entropy thresholding methods both introduce a nonextensive parameter, which is used to describe the long-range correlation between the gray levels of each pixel in the image. This correlation is different from that between neighboring pixels: it is global and covers the entire image. However, the correlation between the gray levels of individual pixels in the image is not global in any situation. For example, we think that the gray levels of the pixels in the target (or background) are related in some infrared image sequences or nondestructive testing images, but it is difficult to determine whether the gray levels in the target and background are also related. Through the comprehensive analysis of the segmentation results of the above test images, it was found that the proposed method is suitable for segmenting images with no obvious correlation between the target and the background, which further verifies the hypothesis of local correlation between the gray levels of each pixel in the image discussed in Sect. 3.

\section{Conclusions}

Tsallis entropy is used to describe long-range interaction within a system in statistical physics. For image processing, this long-range interaction can be understood as the correlation between the gray values of pixels within the image. However, owing to the complexity of natural images, not all images have uniform global correlations. Therefore, we proposed a new thresholding method based on Tsallis cross-entropy by analyzing the information contained in the gray-level histogram of an image and judging the correlation characteristics within. Since the proposed method takes full consideration of the local long-range correlation existing between pixels within the image, the result obtained by the method when segmenting images with no obvious correlation between the target and the background is significantly better than that obtained by the minimum Tsallis cross-entropy thresholding method under the hypothesis of global long-range correlation.

\section{Acknowledgments}

This work was supported by the Climbing Project for Young Teachers of Longyan College (LQ2017002).

\section{References}

1 C. H. Li and C. K. Lee: Pattern Recognit. 26 (1993) 617. https://doi.org/10.1016/0031-3203(93)90115-D

2 A. D. Brink and N. E. Pendock: Pattern Recognit. 29 (1996) 179. https://doi.org/10.1016/0031-3203(95)00066-6

3 N. R. Pal: Pattern Recognit. 29 (1996) 575. https://doi.org/10.1016/0031-3203(95)00111-5

4 J. H. Xue and Y. J. Zhang: Acta Electronica Sinica 27 (1999) 131. http://dx.chinadoi.cn/10.3321\%2fj. issn\%3a0372-2112.1999.10.038

5 Y. G. Tang, Q. Y. Di, X. P. Guan, and F. Liu: Chin. J. Sci. Instrum. 29 (2008) 1868. http://dx.chinadoi. cn/10.3321/j.issn:0254-3087.2008.09.015

6 Y. G. Tang, Q. Y. Di, L. X. Zhao, X. P. Guan, and F. C. Liu: Acta Phys. Sinica 58 (2009) 9. http://dx.chinadoi. $\mathrm{cn} / 10.3321 / \mathrm{j}$.issn:1000-3290.2009.01.002

7 A. L. Porta, G. A. Voth, A. M. Crawford, J. Alexander, and E. Bodenschatz: Nature 409 (2001) 1017. https:// doi.org/10.1038/35059027 
8 B. Liu and J. Goree: Phys. Rev. Lett. 100 (2008) 055003. https://doi.org/10.1103/PhysRevLett.100.055003

9 P. K. Sahoo and G. Arora: Pattern Recognit. Lett. 27 (2006) 520. https://doi.org/10.1016/j.patrec.2005.09.017

10 S. H. Cheng and C. H. Hu: Infrared Laser Eng. 40 (2011) 559. http://dx.chinadoi.cn/10.3969/ j.issn.1007-2276.2011.03.037

11 Y. Q. Wu, Z. Pan, and W. Y. Wu: Opto-Electron. Eng. 35 (2008) 53. http://dx.chinadoi.cn/10.3969/j.issn.1003501X.2008.07.011

12 S. Furuichi, K. Yanagi, and K. Kuriyama: J. Math. Phys. 45 (2004) 4868. https://doi.org/10.1063/1.1805729

13 W. A. Yasnoff, J. K. Mui, and J. W. Bacus: Pattern Recognit. 9 (1977) 217. https://doi.org/10.1016/00313203(77)90006-1

\section{About the Authors}

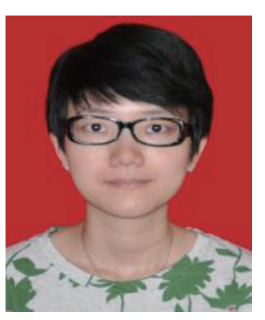

Qian-Qian Lin received her B.S. degree in communication engineering from Huaqiao University in 2010 and her M.S. degree in electronic and communication engineering in 2013. She joined the School of Mathematics and Information Engineering of Longyan University, Longyan, Fujian, China, in 2016. Her main research interests include computer science, computer graphics, and image processing.

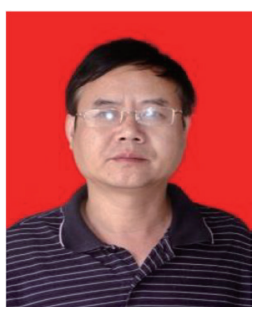

Ling Zhang received his B.S. degree from the College of Mathematics and Informatics of Fujian Normal University, Fujian, China, in 1983 and his M.S. degree from the College of Mathematics and Computer Science of Fuzhou University, Fujian, China, in 2009. He is now the Dean of the Faculty of the School of Mathematics and Information Engineering, Longyan University, Fujian, China. His research interests lie in the fields of Internet of Things, big data, applied mathematics, and AI.

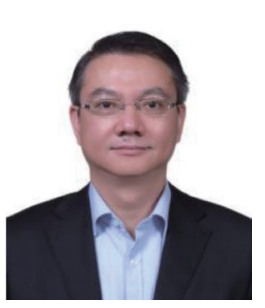

Tean-Shine Mean received his Ph.D. degree from National Central University, Taiwan, in 2001. He was an assistant professor and a director of an eCommerce center and an incubation center of National Kaohsiung University of Science and Technology in 2002-2006. Then, he has been working in various fields of business, including aviation, fast moving consumer goods, information technology, and banking. He is still collaborating with academicians to do research in Fintech.

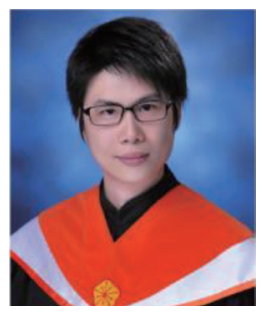

Hsien-Wei Tseng received his B.S. degree in electrical engineering from National Kaohsiung University of Applied Sciences in 2002 and his Ph.D. degree in electrical engineering from Tamkang University in 2010. He joined the School of Information Engineering, Longyan University, Longyan, Fujian, China, in 2017 and now serves as a professor. His main research interests include system design and performance evaluation in wireless communication systems. 
Tung-Lung Wu received his Ph.D. degree from National Formosa University. He joined the School of Mechanical and Automotive Engineering, Zhaoqing University, Zhaoqing, Guangdong, China, in August 2018 and now serves as an associate professor. His main research interests include solar cells, nanomaterials, and computer networks. 